\title{
Soluções eletrolíticas enterais hipotônicas em equinos: efeitos de fontes de energia sobre determinados indicadores do equilíbrio ácido base
}

\author{
Enteral hypotonic electrolyte solutions in horse: effects of energy sources on some indicators of \\ acid - base balance
}

\begin{abstract}
Ana Estela Pessin ${ }^{\mathrm{I} *}$ José Dantas Ribeiro Filho ${ }^{\mathrm{I}}$ Athina Chaves Donner ${ }^{\mathrm{I}}$ Dyego Pimenta Oliveira Antonio de Pádua Lima ${ }^{I}$ Brunna Patrícia Almeida da Fonseca ${ }^{I}$
\end{abstract}

\section{RESUMO}

No presente estudo, foram avaliados os efeitos das soluções eletrolíticas hipotônicas administradas via enteral por sonda nasoesofágica de pequeno calibre em fluxo contínuo sobre o equilíbrio ácido base, lactato plasmático e $\mathrm{pH}$ urinário de equinos. Foram utilizadas seis fêmeas equinas adultas, distribuídas aleatoriamente em três grupos, cada um contendo seis animais, em sistema cross over $6 \times 3$ (seis animais $x$ três tratamentos). Os animais foram submetidos aos tratamentos: SEDext - $5 \mathrm{~g}$ de cloreto de sódio, $0,5 \mathrm{~g}$ de cloreto de potássio, $1 \mathrm{~g}$ de gluconato de cálcio, $200 \mathrm{mg}$ de pidolato de magnésio e $15 \mathrm{~g}$ de dextrose, diluídos em $1.000 \mathrm{~mL}$ de água. Osmolaridade mensurada: $264 \mathrm{mOsmol} / \mathrm{L}$. SEMalt - solução eletrolítica enteral acrescida de $15 \mathrm{~g}$ de maltodextrina diluídos em $1.000 \mathrm{~mL}$ de água. Osmolaridade mensurada 203mOsmol $L^{-1}$. SESaca - solução eletrolítica enteral acrescida de $15 \mathrm{~g}$ de sacarose diluídos em 1.000mL de água. Osmolaridade mensurada: $234 \mathrm{mOsmol} \mathrm{L}^{-1}$. As soluções eletrolíticas foram administradas na dose de $15 \mathrm{~mL}$ $\mathrm{kg}^{-1} \mathrm{~h}^{-1}$, durante 12 horas em fluxo contínuo. Os tratamentos com soluções eletrolíticas enterais, associadas à dextrose $e$ maltodextrina, não alteraram os valores da hemogasometria, do ânion gap, da diferença de íons fortes mensurados e do lactato plasmático, enquanto a administração das três soluções ocasionou diminuição nos valores do $\mathrm{pH}$ urinário. Apesar disso, elas são clinicamente seguras para ser utilizadas em equinos.

Palavras-chave: hidratação, carboidratos, hemogasometria, homeostase.

\section{ABSTRACT}

There were analyzed, in the following study, the effects of electrolytic hypotonic solutions that were administrated using small-caliber nasal esophageal probe with continual flow on acid-base balance, plasma lactate and urinary $\mathrm{pH}$ of horses . Six adult female were distributed in cross over system $6 \times 3$ on a mixed model simultaneously. The animals were distributed in three groups and each of them were managed by the following treatment:
SEDext - $5 \mathrm{~g}$ of sodium chloride, $5 \mathrm{~g}$ of potassium chloride, $1 \mathrm{~g}$ of calcium gluconate, $200 \mathrm{mg}$ of magnesium pidolate, and $15 \mathrm{~g}$ of maltodextrin diluted on a $1.000 \mathrm{~mL}$ of water. The $m$ osmolarity measured was of $264 \mathrm{mOsmol} \mathrm{L^{-1 }}$. SEMalt-enteral electrolyte solution plus $15 \mathrm{~g}$ of maltodextrin diluted on a $1.000 \mathrm{~mL}$ of water. The osmolarity measured was of $203 \mathrm{mOsmol} \mathrm{L}^{-1}$. SESucr - enteral electrolyte solution plus $15 \mathrm{~g}$ of sucrose diluted on a $1,000 \mathrm{~mL}$ of water. The osmolarity measured was of: $234 \mathrm{mOsmol} / \mathrm{L}$. The electrolytic solutions were administered at a dose of $15 \mathrm{ml} \mathrm{kg} \mathrm{k}^{-1}$ $h^{-1}$ for 12 hours. Treatments associated with enteral electrolyte solutions associated to dextrose and maltodextrin did not change the average values of blood gas analysis, anion gap, measured strong ion difference and plasma lactate, while the administration of the three solutions caused a decrease in urinary $\mathrm{pH}$ values. Nevertheless, they are safe clinically to be used in horses.

Key words: fluid therapy, carbohydrates, blood gas analysis, homeostasis.

\section{INTRODUÇÃO}

Alterações hidro-eletrolíticas e ácido base são extremamente comuns em pacientes equinos clínicos e cirúrgicos (CORLEY, 2007). O exercício de longa duração e algumas doenças ou síndromes como diarreias, cólicas, compactações gastrintestinais, obstruções intestinais, peritonite, duodenojejunite proximal e insuficiência renal são capazes de alterar o balanço hidro-eletrolítico e ácido base na espécie equina, sendo a correção desses distúrbios realizada através da administração de soluções eletrolíticas, com o objetivo de recompor a volemia e homeostase (RIBEIRO FILHO et al., 2009).

\footnotetext{
IDepartamento de Veterinária, Universidade Federal de Viçosa (UFV), 36570-000, Viçosa, MG, Brasil. E-mail:anapessin@hotmail.com.
} *Autor para correspondência. 
A tonicidade e composição das soluções eletrolíticas ideais para cada espécie e enfermidade não estão completamente esclarecidas. A solução de hidratação ideal é formulada para repor água e eletrólitos, dependendo da necessidade do paciente, devendo ser modificada com base na avaliação clínica e exames laboratoriais no decorrer do tratamento (RIBEIRO FILHO et al., 2011). Na rotina clínica, são utilizadas soluções eletrolíticas comerciais por via intravenosa, isso porque essa via permite a infusão rápida de grande quantidade de líquido, ocasionando a expansão veloz do volume plasmático, por isso o seu uso é indispensável nos casos de desidratação intensa e choque hipovolêmico. Por sua vez, a administração de soluções eletrolíticas por via enteral é uma alternativa segura e eficaz à via intravenosa em casos de desidratação discreta e moderada (RIBEIRO FILHO et al., 2009).

Ensaios clínicos realizados em crianças e animais de laboratório têm evidenciado resultados superiores na utilização de soluções eletrolíticas enterais hipotônicas, corrigindo os desequilíbrios hidro-eletrolíticos, ácido base e a hipoglicemia mais rapidamente, reduzindo a duração da diarreia e o tempo de internamento dos pacientes (RAUTANEN et al., 1993; NISHINAKA et al., 2004). Em equinos, FARIAS (2010) registrou maior expansão do volume plasmático nos animais tratados com soluções hipotônicas enterais, quando comparados aos tratados com solução isotônica enteral. À exceção do estudo experimental realizado por FARIAS et al. (2011), no qual foram utilizadas soluções eletrolíticas hipotônicas enterais contendo maltodextrina e dextrose como fonte de energia, na maioria das pesquisas realizadas na espécie equina com soluções reidratantes, utilizou-se soluções eletrolíticas isotônicas enterais (ALVES et al., 2005; AVANZA et al., 2009; GOMES et al., 2012).

Açúcares e amido sofrem metabolismo bacteriano no trato gastrointestinal, gerando ácidos orgânicos (ZHANG et al., 2003), consequentemente, podendo ocasionar o aparecimento de acidose. Na espécie equina, o uso de soluções eletrolíticas contendo uma fonte de energia é essencial em alguns pacientes, porém pouco se conhece sobre o efeito de soluções eletrolíticas hipotônicas enterais associadas à maltodextrina, dextrose ou sacarose sobre a homeostase. Por tudo isso, objetivou-se, com o presente ensaio, avaliar os efeitos de soluções eletrolíticas hipotônicas enterais, associadas à sacarose, dextrose ou maltodextrina administradas por sonda nasoesofágica de pequeno calibre, via enteral em fluxo contínuo, durante 12 horas, sobre o equilíbrio ácido base, lactato e pH urinário de equinos.

\section{MATERIAL E MÉTODOS}

Foram utilizados seis equinos hígidos, fêmeas não gestantes, sem raça definida, com

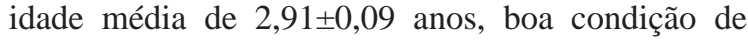
escore corporal, segundo a classificação de SPEIRS (1997), e peso corporal médio de $325 \pm 22 \mathrm{~kg}$ e sem histórico de doença gastrointestinal nos últimos seis meses. Antes do início do experimento, os animais passaram por um período de adaptação de 15 dias e foram submetidos a exame físico, hemograma, perfil bioquímico e exame parasitológico das fezes. Foram alojados em baias individuais e arejadas, com cama de maravalha, limpa e trocada diariamente e foi realizado o controle de ectoparasitas (deltametrina a 0, 025\%) e endoparasitas (praziquantel + ivermectina) após exame físico. A dieta era constituída de feno de tifton 85 (Cynodon sp) à vontade, ração comercial ${ }^{\mathrm{a}}$ concentrada em quantidade equivalente a $1 \%$ do peso corporal, duas vezes/dia, suplemento mineral ${ }^{\mathrm{b}}$, $50 \mathrm{~g}$ $\mathrm{dia}^{-1}$, e água ad libitum.

A distribuição inicial dos animais foi aleatória em três grupos, cada um contendo seis animais (Tabela 1), em sistema cross over 6x3 (seis animais $\mathrm{x}$ três tratamentos), com intervalos entre os períodos de tratamento não menores que sete dias. Os tratamentos foram assim constituídos: grupo SEMalt com solução eletrolítica contendo $5 \mathrm{~g}$ de $\mathrm{NaCl}, 0,5 \mathrm{~g}$ de $\mathrm{KCl}, 1 \mathrm{~g}$ de gluconato de cálcio, 200mg de pidolato de Mg e $15 \mathrm{~g}$ de maltodextrina, diluídos em quantidade suficiente para $1.000 \mathrm{~mL}$ de água (osmolaridade mensuradac: 203mMol L-1); o grupo SEDext recebeu solução eletrolítica contendo $5 \mathrm{~g}$ de $\mathrm{NaCl}, 0,5 \mathrm{~g}$ de $\mathrm{KCl}, 1 \mathrm{~g}$ de gluconato de cálcio, 200mg de pidolato de $\mathrm{Mg}$ e $15 \mathrm{~g}$ de dextrose diluídos por $1.000 \mathrm{~mL}$ de água (osmolaridade mensurada: $264 \mathrm{mMol} \mathrm{L}^{-1}$ ); e, no grupo SESaca, solução eletrolítica contendo $5 \mathrm{~g}$ de $\mathrm{NaCl}, 0,5 \mathrm{~g}$ de $\mathrm{KCl}, 1 \mathrm{~g}$ de gluconato de cálcio, 200mg de pidolato de Mg e 15g de sacarose, diluídos em 1.000mL de água (osmolaridade mensurada: 234mMol L $\mathrm{m}^{-1}$ ). As soluções eletrolíticas hipotônicas enterais foram administradas na dose de $15 \mathrm{~mL} \mathrm{~kg}^{-1} \mathrm{~h}^{-1}$, durante doze horas em fluxo contínuo por sonda nasoesofágicad de pequeno calibre, 5,7mm de diâmetro e 1,5m de comprimento. Durante a fase de hidratação (T0h a T12h), os animais permaneceram em jejum hídrico e alimentar e sem contenção, permanecendo soltos na baia.

Amostras de sangue foram coletadas anaerobicamente após antissepsia para análise 
Tabela 1 - Distribuição dos animais nos tratamentos.

\begin{tabular}{lcc}
\hline Ciclos & Animais & Tratamentos \\
\hline $1^{\circ}$ & 1,2 e 3 & SEMalt, SEDext, SESaca \\
$1^{\circ}$ & 4,5 e 6 & SEMalt, SEDext, SESaca \\
\hline $2^{\circ}$ & 1,2 e 3 & SEDext, SESaca, SEMalt \\
$2^{\circ}$ & 4,5 e 6 & SEDext, SESaca, SEMalt \\
\hline $3^{\circ}$ & 1,2 e 3 & SESaca, SEMAlt, SEDext \\
\hline $3^{\circ}$ & 4,5 e 6 & SESaca, SEMAlt, SEDext \\
\hline
\end{tabular}

SEDext - solução eletrolítica contendo dextrose.

SEMalt - solução eletrolítica contendo maltodextrina.

SESaca - solução eletrolítica contendo sacarose.

hemogasométrica ${ }^{\mathrm{e}}$, por venopunção jugular, em seringas plásticas descartáveis de $3 \mathrm{~mL}$, previamente heparinizadas e acondicionadas em água com gelo, sendo encaminhadas imediatamente para análise. As variáveis mensuradas foram: $\mathrm{pH}_{(\mathrm{v})}$; pressão parcial de oxigênio $-\mathrm{pO}_{2(\mathrm{v})}$; pressão parcial do dióxido de carbono - $\mathrm{pCO}_{2(\mathrm{v})}$; concentração total do dióxido de carbono - $\mathrm{tCO}_{2(\mathrm{v})}^{2(\mathrm{v})}$; concentração de bicarbonato - $\mathrm{CHCO}_{3(\mathrm{v})}^{-}$; concentração de bases tituláveis cBase ${ }_{(v)}$; e saturação de oxigênio $-\mathrm{sO}_{2(\mathrm{v})}$. Por sua vez, o soro foi obtido de amostras sanguíneas colhidas em frascos Vacutainer sem anticoagulante para determinação do sódio ${ }^{\mathrm{f}}$, potássio ${ }^{\mathrm{f}}$ e cloreto $^{\mathrm{g}}$. A coleta de sangue foi realizada também utilizando frascos Vacutainer com fluoreto de sódio para obtenção de plasma para mensuração do lactatog. $\mathrm{O} \mathrm{pH}$ urinário foi determinado por fita reagente ${ }^{\mathrm{h}}$. O valor dos ânions gap (AG) foi obtido adotandose a fórmula: AG $\left.(\mathrm{mEq} \mathrm{L})^{-1}\right)=\left(\mathrm{Na}^{+}+\mathrm{K}^{+}\right)-\left(\mathrm{Cl}^{-}+\right.$ $\mathrm{HCO}_{3}^{-}$), enquanto a diferença de íons fortes foram mensurados pela fórmula: $\operatorname{DIFm~}\left(\mathrm{mEq} \mathrm{L} \mathrm{L}^{-1}\right)=$ $\left(\mathrm{Na}^{+}+\mathrm{K}^{+}\right)-\left(\mathrm{Cl}^{-}\right)$. As análises laboratoriais foram realizadas nos intervalos: T0h (imediatamente antes da hidratação), T6h (seis horas de hidratação), T12h (término da hidratação) e T24h (doze horas após o término da hidratação).

Os dados obtidos foram submetidos ao teste ANOVA (análise de variância), baseada em planejamento de medidas repetidas, ou seja, foi avaliado cada tratamento em vários tempos de acompanhamento. Além de avaliar a influência do tratamento foi analisada a influência do tempo, bem como a interação entre o tratamento e o tempo. Quando a análise foi significativa para um ou mais fatores, foi utilizado o teste de Tukey (SAEG, 2007). Todas as análises foram consideradas significativas quando $\mathrm{P}<0,05$.

\section{RESULTADOS E DISCUSSÃO}

Durante as primeiras seis horas de hidratação (T6h), constatou-se discreta diminuição nos valores do $\mathrm{pH}$ sanguíneo nos animais dos três tratamentos, porém, apenas no tratamento SESaca o decréscimo foi significativo $(\mathrm{P}<0,05)$. Apesar da redução significativa, os valores mantiveram-se na faixa de referência para a espécie equina (KANEKO et al., 2008), tornando-os sem significado clínico. Além disso, apesar de os animais continuarem a receber a solução eletrolítica (SESaca), no T12h, eles retornaram a níveis semelhantes ao T0h, ratificando que a alteração mínima observada no valor médio do pH sanguíneo, ocasionada pela SESaca no T6h, é desprovida de relevância clínica (Tabela 2).

Os valores médios da concentração de base (cBase) apresentaram comportamento semelhante aos do $\mathrm{pH}$, ou seja, houve diminuição durante a fase de hidratação (T6h e T12h) nos animais de todos os tratamentos (Tabela 2). Apenas os animais que receberam a solução eletrolítica contendo sacarose (SESaca) apresentaram decréscimo significativo $(\mathrm{P}<0,05)$ nos valores de cBase em T6h $(\mathrm{P}<0,05)$. Apesar da redução da cBase nos animais de todos os tratamentos, os valores também permaneceram na faixa de referência (KANEKO et al., 2008).

Esse decréscimo, possivelmente, ocorreu devido ao efeito da fonte de energia contida nas soluções eletrolíticas, pois, segundo ZHANG et al. (2003), a ingestão de açúcares e amido sofre metabolismo bacteriano no trato gastrointestinal, gerando ácidos orgânicos, consequentemente, podendo ocasionar o aparecimento de acidose. Como no equino a passagem da ingesta pelo intestino delgado é rápida (ECKE et al. 1998), as soluções eletrolíticas chegam ao intestino grosso contendo quantidades 
Tabela 2 - Médias e desvios padrão do $\mathrm{pH}_{(\mathrm{v})}, \mathrm{cHCO}_{3 \mathrm{v}}^{-}$, $\mathrm{tCO}_{2 \mathrm{v}}, \mathrm{cBase}_{\mathrm{v}}, \mathrm{pCO}_{2 \mathrm{v}}, \mathrm{pO}_{2 \mathrm{v}}$ e $\mathrm{sO}_{2 \mathrm{v}}$ de equinos tratados com soluções eletrolíticas enterais, contendo maltodextrina (SEMalt), dextrose (SEDext) ou sacarose (SESaca), administradas por sonda nasoesofágica de pequeno calibre e fluxo contínuo $\left(15 \mathrm{~mL} \mathrm{~kg}^{-1} \mathrm{~h}^{-1} 12 \mathrm{~h}^{-1}\right)$.

\begin{tabular}{|c|c|c|c|c|}
\hline Tratamentos & T0h & T6h & $\mathrm{T} 12 \mathrm{~h}$ & T24h \\
\hline & ----------------' & ---------------- & -------------------- & 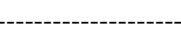 \\
\hline SEMalt & $7,39 \pm 0,008^{\mathrm{Aa}}$ & $7,37 \pm 0,02^{\mathrm{Aa}}$ & $7,37 \pm 0,03^{\mathrm{Aa}}$ & $7,39 \pm 0,02^{\mathrm{Aa}}$ \\
\hline SEDext & $7,40 \pm 0,01^{\mathrm{Aa}}$ & $7,37 \pm 0,02^{\mathrm{Aa}}$ & $7,37 \pm 0,02^{\mathrm{Aa}}$ & $7,38 \pm 0,02^{\mathrm{Aa}}$ \\
\hline SESaca & $7,39 \pm 0,01^{\mathrm{Aa}}$ & $7,35 \pm 0,01^{\mathrm{Ab}}$ & $7,39 \pm 0,02^{\text {Aa }}$ & $7,40 \pm 0,02^{\mathrm{Aa}}$ \\
\hline & & ---------------ch & ) ---------------- & \\
\hline SEMalt & $29,75 \pm 1,63^{\text {Аа }}$ & $27,13 \pm 1,96^{\mathrm{Aa}}$ & $26,65 \pm 2,17^{\text {Аа }}$ & $27,28 \pm 2,24^{\mathrm{Aa}}$ \\
\hline SEDext & $29,53 \pm 3,01^{\mathrm{Aa}}$ & $27,01 \pm 2,12^{\mathrm{Aa}}$ & $26,18 \pm 1,12^{\mathrm{Aa}}$ & $27,23 \pm 1,59^{\mathrm{Aa}}$ \\
\hline SESaca & $28,48 \pm 2,94^{\mathrm{Aa}}$ & $25,70 \pm 2,16^{\mathrm{Aa}}$ & $26,33 \pm 1,77^{\text {Аа }}$ & $28,96 \pm 1,72^{\mathrm{Aa}}$ \\
\hline SEMalt & $31,26 \pm 1,70^{\mathrm{Aa}}$ & $28,15 \pm 2,99^{\mathrm{Aa}}$ & $28,05 \pm 2,24^{\mathrm{Aa}}$ & $28,70 \pm 2,33^{\text {Аa }}$ \\
\hline SEDext & $30,98 \pm 3,12^{\mathrm{Aa}}$ & $28,46 \pm 2,20^{\mathrm{Aa}}$ & $27,53 \pm 1,17^{\text {Аа }}$ & $28,70 \pm 1,59^{\mathrm{Aa}}$ \\
\hline SESaca & $29,91 \pm 3,08^{\text {Аa }}$ & $27,11 \pm 2,25^{\mathrm{Aa}}$ & $27,65 \pm 1,80^{\text {Аа }}$ & $30,35 \pm 1,70^{\mathrm{Aa}}$ \\
\hline SEMalt & $4,50 \pm 1,50^{\mathrm{Aa}}$ & $1,95 \pm 1,77^{\text {Аа }}$ & $1,73 \pm 2,22^{\mathrm{Aa}}$ & $2,48 \pm 1,95^{\mathrm{Aa}}$ \\
\hline SEDext & $4,53 \pm 2,62^{\mathrm{Aa}}$ & $1,85 \pm 2,08^{\mathrm{Aa}}$ & $1,46 \pm 1,07^{\mathrm{Aa}}$ & $2,23 \pm 1,58^{\mathrm{Aa}}$ \\
\hline SESaca & $3,50 \pm 2,47^{\text {Aab }}$ & $0,38 \pm 1,97^{\mathrm{Ab}}$ & $1,80 \pm 1,90^{\mathrm{Aab}}$ & $4,16 \pm 1,99^{\text {Аа }}$ \\
\hline SEMalt & $50,00 \pm 1,78^{\mathrm{Aa}}$ & $47,91 \pm 3,20^{\mathrm{Aa}}$ & $47,36 \pm 3,67^{\text {Аа }}$ & $45,71 \pm 3,89^{\mathrm{Aa}}$ \\
\hline SEDext & $48,58 \pm 3,72^{\text {Аа }}$ & $47,51 \pm 2,25^{\mathrm{Aa}}$ & $45,76 \pm 2,77^{\text {Аа }}$ & $47,25 \pm 4,33^{\mathrm{Aa}}$ \\
\hline SESaca & $47,96 \pm 5,39^{\mathrm{Aa}}$ & $47,08 \pm 3,80^{\mathrm{Aa}}$ & $44,83 \pm 2,22^{\mathrm{Aa}}$ & $47,45 \pm 2,64^{\mathrm{Aa}}$ \\
\hline SEMalt & $40,78 \pm 4,71^{\mathrm{Aa}}$ & $44,96 \pm 4,08^{\mathrm{Aa}}$ & $47,96 \pm 4,34^{\mathrm{Aa}}$ & $42,93 \pm 5,84^{\mathrm{Aa}}$ \\
\hline SEDext & $39,45 \pm 2,30^{\mathrm{Aa}}$ & $44,30 \pm 3,81^{\mathrm{Aa}}$ & $46,68 \pm 1,49^{\text {Аа }}$ & $46,35 \pm 11,76^{\mathrm{Aa}}$ \\
\hline SESaca & $40,90 \pm 5,36^{\mathrm{Aa}}$ & $46,53 \pm 7,70^{\mathrm{Aa}}$ & $47,95 \pm 5,74^{\mathrm{Aa}}$ & $43,38 \pm 9,10^{\mathrm{Aa}}$ \\
\hline SEMalt & $73,41 \pm 6,46^{\mathrm{Aa}}$ & $78,06 \pm 4,81^{\mathrm{Aa}}$ & $80,01 \pm 3,40^{\mathrm{Aa}}$ & $75,61 \pm 5,98^{\mathrm{Aa}}$ \\
\hline SEDext & $72,38 \pm 3,91^{\text {Аа }}$ & $77,40 \pm 4,75^{\mathrm{Aa}}$ & $79,10 \pm 2,97^{\mathrm{Aa}}$ & $77,70 \pm 7,47^{\text {Аа }}$ \\
\hline SESaca & $72,93 \pm 6,57^{\mathrm{Aa}}$ & $77,76 \pm 8,61^{\mathrm{Aa}}$ & $80,51 \pm 3,15^{\mathrm{Aa}}$ & $75,43 \pm 8,85^{\mathrm{Aa}}$ \\
\hline
\end{tabular}

Análise de variância (medidas repetidas).

Valores médios seguidos por letra maiúsculas diferentes na mesma coluna ou por letras minúsculas diferentes na mesma linha diferem estatisticamente entre si $(\mathrm{P}<0,05)$ pelo teste de Tukey.

T0h (imediatamente antes da hidratação), T6h (seis horas de hidratação), T12h (término da hidratação) e T24h (doze horas após o término da hidratação).

$\mathrm{cHCO}_{3(\mathrm{v})}^{-}$- concentração de bicarbonato, $\mathrm{tCO}_{2 \mathrm{v}}$ - concentração total do dióxido de carbono, cBase $(\mathrm{v})$ - concentração de bases tituláveis, $\mathrm{pCO}_{2(\mathrm{v})}$ - pressão parcial do dióxido de carbono, $\mathrm{pO}_{2 \mathrm{v}}$ - pressão parcial de oxigênio, $\mathrm{sO}_{2(\mathrm{v})}$ - saturação de oxigênio.

significativas de carboidratos, desencadeando ação fermentativa da microbiota, gerando o aparecimento de substâncias ácidas, as quais, ao serem absorvidas, determinam diminuição nos valores do $\mathrm{pH}$ e da cBase sanguíneos. FARIAS et al. (2011) também relataram discreta diminuição do $\mathrm{pH}$ e da cBase sanguíneos após seis horas de hidratação e posterior acréscimo ao término da $12^{a}$ hora, utilizando solução eletrolítica enteral, contendo, como fonte de energia, a dextrose e maltodextrina. Da mesma forma que o presente ensaio, os valores do $\mathrm{pH}$ e da cBase obtidos pelos referidos autores, apesar da diminuição, também mantiveram-se na faixa de referência para animais da espécie equina (KANEKO et al., 2008).

Pelo exposto, todas as soluções eletrolíticas utilizadas nos animais tinham potencial acidificante discreto. Entretanto, nesses casos, o organismo recorre a mecanismos de compensação e, dentre eles, os rins exercem papel de destaque, através da eliminação de íons $\mathrm{H}^{+}$pela urina. A partir do T6h, percebe-se que o organismo animal começou a utilizá-los, o qual é expresso pelo aumento dos valores do $\mathrm{pH}$ e cBase sanguíneos. Esse mecanismo foi detectado pela presença de acidúria nos animais 
de todos os tratamentos até a sexta hora (T6h) (Tabela 3). A partir de T6h, os valores do pH urinário começaram a aumentar, embora os animais ainda estivessem recebendo as soluções eletrolíticas, o que ocorreu até a 12a hora (T12h). Esperava-se que o $\mathrm{pH}$ urinário continuasse decrescendo ou pelo menos que se estabilizasse. Entretanto, isso não ocorreu, possivelmente foi ocasionado pela produção excessiva de urina, decorrente da hidratação, a qual determinou a diluição dos íons $\mathrm{H}^{+}$com consequente aumento do pH urinário. Esses resultados demonstraram que os rins atuaram de maneira eficaz na manutenção da homeostase corporal, impedindo alterações no equilíbrio ácido base.

À exceção dos animais do grupo SESaca, que apresentaram discreto aumento nos valores de lactato na fase final de hidratação $(\mathrm{P}>0,05)$, nas soluções eletrolíticas contendo dextrose (SEDext) e maltodextrina (SEMalt), houve decréscimo nos valores do lactato plasmático em T12h (Tabela 3). Um efeito colateral importante, quando se utilizam soluções eletrolíticas contendo fontes de energia, é a possibilidade de essas substâncias predisporem o aparecimento de acidose nos animais, porém os resultados encontrados no presente estudo experimental demonstram que a maltodextrina, a dextrose e a sacarose, utilizadas na dose de $15 \mathrm{~g}$ por litro de solução, não ocasionaram o aparecimento do referido efeito adverso, o que as torna segura para o uso na hidratação enteral em equinos. Esse achado confirma a afirmação anterior, de que as substâncias utilizadas como fonte de energia no presente estudo experimental não são acidificantes.

O bicarbonato $\left(\mathrm{CHCO}_{3}^{-}\right.$) e a tCO $\mathrm{CO}_{2}$ (Tabela 2) não apresentaram diferenças nos valores médios entre os tratamentos e nos tratamentos ao longo do tempo $(\mathrm{P}>0,05)$, confirmando os relatos de SOSA LÉON et al. (1995) e FARIAS et al. (2011), que também não registraram alterações significativas nessas variáveis, ressaltando-se que, embora os referidos autores tenham utilizado soluções eletrolíticas enterais em equinos, elas tinham composição diferentes das utilizadas no presente ensaio. A ausência de significado nos valores do $\mathrm{CHCO}_{3}^{-}$e da $\mathrm{tCO}_{2}(\mathrm{P}>0,05)$ ratifica que a tendência à acidificação apresentada pelos animais do SESaca foi discreta, consequentemente, desprovida de significado clínico.

A pressão parcial de oxigênio $\left(\mathrm{pO}_{2}\right)$ e a saturação de oxigênio no sangue $\left(\mathrm{sO}_{2}\right)$ também não apresentaram variações entre os valores médios entre tratamentos e nos tratamentos ao longo do tempo ( $\mathrm{P}>0,05)$ (Tabela 2). Resultados semelhantes foram relatados por GOMES (2010) e FARIAS et al. (2011).
Os valores de referência do ânion gap (AG) para espécie equina variam entre 5 a 16,2 mEq $\mathrm{L}^{-1}$ (WHITEHAIR et al., 1995; CARLSON, 2008). Como pode ser verificado na tabela 3 , não se observou diferença nos valores do AG entre os tratamentos testados e nos tratamentos ao longo da fase experimental $(\mathrm{P}>0,05)$. Esse achado também reforça que as soluções eletrolíticas testadas na presente pesquisa não são acidificantes, pois, como relatou CARLSON (2008) e DiBARTOLA (2012), valores superiores à faixa de referência indicam acidose metabólica e, como pode ser constatado na tabela 3, isso não ocorreu. Por sua vez, a diferença de íons fortes mensurados (DIFm) também não apresentou diferença $(P>0,05)$ entre tratamentos, tampouco nos tratamentos ao longo da fase experimental (Tabela 3), ratificando a conclusão obtida através dos valores do AG. Segundo LINDIGER (2004), valores da DIF superiores à faixa de referência (37-43 mMol L $\left.{ }^{-1}\right)$ indicam alcalose metabólica e os inferiores indicam acidose metabólica. Entretanto, observando-se os valores da DIFm do presente ensaio, percebe-se que são menores que os citados como fisiológicos por LINDIGER (2004), levando à ponderação feita por FARIAS et al. (2011) sobre tabelas com valores de referência que, algumas vezes, não expressam a realidade de uma raça, de uma faixa etária ou de uma determinada região.

\section{CONCLUSÃO}

Os tratamentos com soluções eletrolíticas enterais hipotônicas, associadas à dextrose ou maltodextrina, administradas por sonda nasoesofágica de pequeno calibre e fluxo contínuo $\left(15 \mathrm{~mL} \mathrm{~kg} \mathrm{~kg}^{-1}\right)$ durante doze horas, não alteraram os valores da hemogasometria, do ânion gap, do DIF e do lactato plasmático. Porém, a administração das três soluções eletrolíticas ocasionou o aparecimento de acidúria. Por não causar alteração no equilíbrio ácido base, as soluções eletrolíticas enterais hipotônicas, acrescidas de dextrose e maltodextrina podem ser consideradas um grande auxílio na hidratação de equinos. Por ocasionar apenas discreta alteração no valor do $\mathrm{pH}$ sanguíneo, a solução eletrolítica enteral hipotônica, contendo sacarose, pode também ser utilizada nos animais da referida espécie.

\section{FONTES DE AQUISIÇÃO}

\footnotetext{
a - Ração SOMA Equinos 12 - Lindo Vale - Rio Pomba - Minas Gerais.

b - Guabiphos Centauro 80\% - Campinas - SP.

c - Advanced Micro-Osmometer Model 3320 - Advanced Instruments, INC. - Massachusetts - USA.
} 
Tabela 3 - Médias e desvios padrão do pH urinário, lactato, anion gap e DIFm, de equinos tratados com soluções eletrolíticas enterais, contendo maltodextrina (SEMalt), dextrose (SEDext) ou sacarose (SESaca), administradas por sonda nasoesofágica de pequeno calibre e fluxo contínuo $\left(15 \mathrm{~mL} \mathrm{~kg}^{-1} \mathrm{~h}^{-1} 12 \mathrm{~h}^{-1}\right)$.

\begin{tabular}{|c|c|c|c|c|}
\hline Tratamentos & TOh & T6h & $\mathrm{T} 12 \mathrm{~h}$ & T24h \\
\hline & \multicolumn{4}{|c|}{----------------------------------------------------pH urinário------------------------------------------------------------------ } \\
\hline SEMalt & $7,66 \pm 0,51^{\text {Aa }}$ & $5,66 \pm 1,03^{\mathrm{Ac}}$ & $6,16 \pm 0,75^{\mathrm{Abc}}$ & $7,33 \pm 0,51^{\mathrm{ABab}}$ \\
\hline SEDext & $7,50 \pm 0,54^{\text {Aa }}$ & $6,08 \pm 0,91^{\mathrm{Ab}}$ & $6,58 \pm 0,73^{\text {Aab }}$ & $6,75 \pm 0,41^{\text {Bab }}$ \\
\hline \multirow[t]{2}{*}{ SESaca } & $7,33 \pm 0,75^{\mathrm{Aa}}$ & $5,41 \pm 0,66^{\mathrm{Ab}}$ & $6,66 \pm 0,75^{\text {Аа }}$ & $7,50 \pm 0,54^{\mathrm{Aa}}$ \\
\hline & & ---------------- & $\left.\mathrm{dL}^{-1}\right)$ & 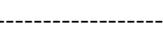 \\
\hline SEMalt & $4,65 \pm 1,15^{\mathrm{Aa}}$ & $5,10 \pm 1,82^{\mathrm{Aa}}$ & $4,05 \pm 0,79^{\mathrm{Aa}}$ & $5,15 \pm 2,25^{\mathrm{Aa}}$ \\
\hline SEDext & $4,35 \pm 1,22^{\mathrm{Aa}}$ & $4,01 \pm 0,78^{\text {Aab }}$ & $2,93 \pm 0,50^{\mathrm{Ab}}$ & $3,01 \pm 0,76^{\text {Aab }}$ \\
\hline \multirow[t]{2}{*}{ SESaca } & $4,38 \pm 0,76^{\mathrm{Aa}}$ & $6,01 \pm 1,80^{\mathrm{Aa}}$ & $4,81 \pm 2,20^{\mathrm{Aa}}$ & $3,45 \pm 0,71^{\mathrm{Aa}}$ \\
\hline & & ----------Anion & $\left.\mathrm{L}^{-1}\right)$ & \\
\hline SEDext & $8,78 \pm 2,48^{\mathrm{Aa}}$ & $10,83 \pm 3,03^{\mathrm{Aa}}$ & $8,78 \pm 3,93^{\mathrm{Aa}}$ & $7,35 \pm 8,23^{\mathrm{Aa}}$ \\
\hline SEMalt & $8,00 \pm 5,82^{\mathrm{Aa}}$ & $9,93 \pm 6,69^{\mathrm{Aa}}$ & $9,01 \pm 5,89^{\text {Аа }}$ & $12,78 \pm 2,98^{\mathrm{Aa}}$ \\
\hline \multirow[t]{2}{*}{ SESaca } & $10,91 \pm 2,11^{\mathrm{Aa}}$ & $12,38 \pm 2,77^{\mathrm{Aa}}$ & $11,38 \pm 4,73^{\mathrm{Aa}}$ & $8,15 \pm 2,90^{\mathrm{Aa}}$ \\
\hline & & ---------DIF & |---------------- & 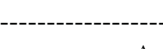 \\
\hline SEDext & $31,45 \pm 2,41^{\mathrm{Aa}}$ & $31,66 \pm 2,18^{\text {Аa }}$ & $29,48 \pm 3,31^{\mathrm{Aa}}$ & $28,18 \pm 7,21^{\mathrm{Aa}}$ \\
\hline SEMalt & $30,73 \pm 4,38^{\mathrm{Aa}}$ & $26,36 \pm 3,79^{\mathrm{Aa}}$ & $25,15 \pm 4,17^{\mathrm{Aa}}$ & $33,55 \pm 4,74^{\mathrm{Aa}}$ \\
\hline SESaca & $29,88 \pm 3,98^{\mathrm{Aa}}$ & $26,88 \pm 3,80^{\text {Aa }}$ & $27,18 \pm 3,91^{\mathrm{Aa}}$ & $30,83 \pm 1,89$ \\
\hline
\end{tabular}

Análise de variância (medidas repetidas).

Valores médios seguidos por letra maiúsculas diferentes na mesma coluna ou por letras minúsculas diferentes na mesma linha diferem estatisticamente entre si $(\mathrm{P}<0,05)$ pelo teste de Tukey.

T0h (imediatamente antes da hidratação), T6h (seis horas de hidratação), T12h (término da hidratação) e T24h (doze horas após o término da hidratação).

DIFm - diferença de íons fortes mensurados.

d - Sonda uretral para equino macho - Provar - Produtos Veterinários - Vila Alpina - SP.

e - ABL80 FLEX - Radiometer Copenhagen.

f - Fotômetro de Chama - B462 - Micronal S/A - São Paulo - SP. g - Aparelho automático de bioquímica HumaStar 300 - Human. Distribuído por in vitro Diagnostica Ltda. Itabira - MG.

h - Fita reagente para urinálise - Combina 115 - Human Wesbaden - Alemanha.

\section{COMITÊ DE ÉTICA}

Artigo submetido à Comissão de Ética e Biossegurança do DVT-UFV. Protocolo 198/2011.

\section{REFERÊNCIAS}

ALVES, G. E. S. et al. Tratamento da compactação experimental do cólon maior em equinos: resultados de laboratório e exames bioquímicos. Arquivo Brasileiro de Medicina Veterinária e Zootecnia, v.57, p.281-287, 2005. Disponível em: <http://www.scielo.br/scielo.php?pid=S010209352005000300001\&script=sci_arttext $>$. Acesso em: 20 nov. 2012. doi. 10.1590/S0102-09352005000300001.

AVANZA, M.F.B. et al. Hidratação enteral em equinos - solução eletrolítica associada ou não à glicose, à maltodextrina e ao sulfato de magnésio: resultados de laboratório. Ciência Rural, v.39, p.1126-1133, 2009. Disponível em: <http://www.scielo.br/scielo. php?script=sci_arttext\&pid $>$. Acesso em: 10 mar. 2010. doi: 10.1590/S0103-84782009005000021.
CARLSON, G.P. Fluid, electrolyte and acid-base balance. In: KANEKO, J.J. et al. Clinical biochemistry domestic animals. 6.ed. London: Elsevier, 2008. Cap.17, p.529-559.

CORLEY, K.T.T. Treating electrolyte abnormalities in colic patients. Compendium of Continuing Education for the Practicing Veterinarian, v.2, n .1, p.16-20, 2007.

DIBARTOLA, S.P. Disorders of sodium and water: hypernatremia and hyponatremia. In: Fluid, electrolyte, and acid-base disorders in small animal practice. 4.ed. Missouri: Elsevier, 2012. Cap.5, p.92-118.

ECKE, P. et al. Induced diarrhoea in horses. Part 2: response to administration of an oral rehydratation solution. Veterinary Journal, v.155, n.2, p.161-170, 1998.

FARIAS, S.K. Efeitos de soluções eletrolíticas associadas ou não à dextrose, maltodextrina e propionato de cálcio administradas por via enteral sobre parâmetros clínicos e laboratoriais de equinos. 2010. 74f. Dissertação (Mestrado em Medicina Veterinária) - Universidade Federal de Viçosa, Viçosa, MG.

FARIAS, S.K. et al. Hemogasometria e ânion gap em equinos tratados com soluções eletrolíticas enterais. Ciência Rural, v.41, n.9, p.1587-1592, 2011. Disponível em: <http://www.scielo. br/scielo.php?pid=S0103-84782011000900016\&script $=$ sci arttext>. Acesso em: 25 de set. 2011. doi: 10.1590/S010384782011000900016 . 
GOMES, C.L.N. Efeitos do polietilenoglicol (PEG 3350) e soluções poliônicas administradas por via enteral e intravenosa em equinos. 2010. 130f. Tese (Doutorado em Medicina Veterinária) - Universidade Federal de Viçosa, Viçosa, MG.

GOMES, C.L.N. et al. Efeitos do polietilenoglicol 3350 e de soluções acidificantes e alcalinizantes sobre o perfil bioquímico de éguas hígidas. Arquivo Brasileiro de Medicina Veterinária e Zootecnia, v.64, p.817-826, 2012. Disponível em: <http://www.scielo.br/scielo.php?pid=S010209352012000400005\&script=sci_arttext $>$. Acesso em: 20 nov. 2012. doi: 10.1590/S0102-09352012000400005.

KANEKO, J.J. et al. Clinical biochemistry of domestic animals. 6.ed. San Diego: Academic, 2008. 932p.

LINDGER, M.I. Acid-base physiology during exercise and in response to training. In: HINCHCLIFF, K.W. et al. Equine sports medicine and surgery. Saunders: London, 2004. Cap.39, p.872-936.

NISHINAKA, D. et al. Water and electrolyte absorption from hypotonic oral rehydration solution in rat small intestine and colon. Pediatrics International, v.46, p.315-321, 2004.

RAUTANEN, T. et al. Clinical experience with a hypotonic oral rehydration solution in acute diarrhoea. Acta Paediatrica, v.82, p.52-54, 1993.

RIBEIRO FILHO, J.D. et al. Hidratação enteral em bovinos: avaliação de soluções eletrolíticas isotônicas administradas por sonda nasogástrica em fluxo contínuo. Ciência Rural, v.41, n.2, p.285-290, 2011. Disponível em: <http://www.scielo.br/scielo. php?script=sci_arttext\&pid=S010384782011000200017\&lng=pt \&nrm=iso >. Acesso em: 01 maio, 2011. doi: org/10.1590/S010384782011005000012 .

RIBEIRO FILHO, J.D. et. al. Hidratação enteral em ruminantes e equídeos. Eficiência com menor custo. Revista do Conselho Federal de Medicina Veterinária. n.48, p.63-67, 2009.

SAEG. Sistema para análises estatísticas. Versão 9.1. Viçosa: UFV, Fundação Arthur Bernardes, 2007. 301p.

SOSA LEON, L.A. et al. The effects of tonicity, glucose concentration e temperature of an oral rehydration solution on its absorption and elimination. Equine Veterinary Journal, Suppl, v.20, p.140-146, 1995.

SPEIRS, V.C. Exame clínico de equinos. Artes Médicas Sul, 1997. 366p.

WHITEHAIR, K.J. et al. Clinical applications on quantitative acid-base chemistry. Journal Veterinary Internal Medicine, v.9, n.1, p.1-11, 1995

ZHANG, D.L. et al. D-lactic acidosis secondary to short bowel syndrome. PostGraduate Medical Journal, n.79, p.110-112, 2003. Disponível em: <http://pmj.bmj.com/content/79/928/110. full>. Acesso em: 25 out. 2011. doi:10.1136/pmj.79.928.110. 